\title{
Efecto analgésico profiláctico del paracetamol e ibuprofeno en la extracción de piezas dentarias en niños
}

\section{Prophylactic analgesic effect of paracetamol and ibuprofen in the extraction of teeth in children}

\author{
Mery Cecilia Paccini Torres ${ }^{1, a}$, Rafael Morales Vadillo,a,ab
}

\section{RESUMEN}

\begin{abstract}
Objetivo: Evaluar el efecto analgésico profiláctico del Paracetamol y el lbuprofeno administrados antes de la extracción de piezas deciduas en niños. Material y método: Se evaluaron 60 niños de 6 a 8 años de edad, que acudieron a la atención en el Centro Odontológico de la Facultad de Odontología de la Universidad de San Martín de Porres, los cuales fueron distribuidos en tres grupos, cada uno de 20 niños: grupo Paracetamol, grupo lbuprofeno y el grupo control. En los dos primeros se les administró, vía oral, 30 minutos antes de la cirugía el fármaco correspondiente (paracetamol $15 \mathrm{mg} / \mathrm{kg} 0$ ibuprofeno $10 \mathrm{mg} / \mathrm{kg}$ ). Fueron evaluadas las siguientes variables: presencia de dolor, tiempo de presencia de dolor, intensidad de dolor, y tiempo transcurrido para analgesia de rescate. Resultados: Se encontraron diferencias significativas en la variable presencia de dolor y en el tiempo de presencia de dolor entre los 3 grupos. No hubo significancia entre las variables estudiadas entre los dos grupos con fármacos. Conclusiones: La profilaxis analgésica es una alternativa eficaz frente al tratamiento convencional farmacológico posquirúrgico. El lbuprofeno y el Paracetamol son medicamentos con eficacia similar para este fin, a pesar de presentar diferente acción farmacológica.
\end{abstract}

Palabras Clave: Paracetamol, Ibuprofeno, Profilaxis analgésica. (Fuente: DeCS BIREME)

\section{ABSTRACT}

Objective: To evaluate the prophylactic analgesic effect of paracetamol and ibuprofen administered before the extraction of deciduous teeth in children. Materials and methods: Sixty children of 6 to 8 years of age were evaluated and attended the Dental Center of the Faculty of Dentistry of the University of San Martín de Porres, which were distributed in three groups, each of 20 children: group paracetamol, ibuprofen group and the control group. In the first two, the corresponding drug was administered orally 30 minutes before surgery (paracetamol $15 \mathrm{mg} / \mathrm{kg}$ or lbuprofen $10 \mathrm{mg} / \mathrm{kg}$ ). The following variables were evaluated: presence of pain, time of presence of pain, intensity of pain, and time elapsed for rescue analgesia. Results: Significant differences were found in the presence of pain variable and in the time of presence of pain among the 3 groups. There was no significance between the variables studied between the two groups with drugs. Conclusions: Analgesic prophylaxis is an effective alternative to conventional post-surgical pharmacological treatment. Ibuprofen and Paracetamol are drugs with similar efficacy for this purpose, despite presenting different pharmacological action.

Key words: Paracetamol, Ibuprofen, Analgesic prophylaxis. (Source: MeSH NLM)

Recibido: 16 de enero de 2019

Aprobado: 29 de marzo de 2018

Publicado:05 Abril 2019

1. Universidad Nacional Mayor de San Marcos

2. Universidad de San Martin de Porres. Lima, Perú.

a Magister, especialista

b Doctor en Educación

Correspondencia:

Mery Cecilia Paccini Torres

Correo electrónico: merypacc@yahoo.com

Citar como: Paccini Torres M, Morales Vadillo R. Efecto analgésico profiláctico del paracetamol e ibuprofeno en la extracción de piezas dentarias en niños. KIRU. 2019; 16(2): 61 - 68. https://doi.org/10.24265/kiru.2019.v16n2.02
Este es un artículo Open Access distribuido bajo la licencia Creative Commons Atribución-NoComercial-

Compartirlgual 4.0 


\section{INTRODUCCIÓN}

El ejercicio de la Odontopediatría plantea retos adicionales y diferentes a los que se manejan cuando se atiende a pacientes adultos. Uno de los principales miedos que enfrenta el niño antes, durante y después de la consulta es la presencia de dolor, definido como "una experiencia sensorial o emocional desagradable asociada a un daño real o potencial en un tejido, o descrito en términos de dicho daño y que incluye una serie de conductas visibles $y / 0$ audibles que pueden ser modificadas por el aprendizaje" (Asociación Internacional para el Estudio del Dolor, IASP) ${ }^{(1,2)}$.

La mayor parte de estudios al respecto enfatizan el valor del uso de analgésicos o antiinflamatorios no esteroideos (AINES) una vez que el dolor se ha desencadenado, creando en algunos casos, esto último, experiencias negativas en el subconsciente del niño, lo que hace que se genere un rechazo a la atención estomatológica. Los estudios sobre profilaxis analgésica en odontología están diseñados y dirigidos a la población adulta, existiendo un vacío en cuanto a su investigación en niños. Los fármacos propuestos son los más empleados y conocidos en la población infantil, habiendo demostrado un bajo perfil de reacciones adversas medicamentosas y efectos colaterales, por lo que su comparación en el esquema terapéutico que se propone es muy segura.

El objetivo de este estudio fue evaluar el efecto analgésico profiláctico del Paracetamol y el Ibuprofeno administrados antes de la extracción de piezas deciduas en niños.

\section{MATERIALES Y METODOS}

La muestra estuvo conformada por 60 pacientes niños de ambos sexos, de 6 a 8 años que acudieron al
Centro Odontológico de la Universidad de San Martin de Porres, quienes cumplieron los criterios de inclusión establecidos. Fueron divididos de manera aleatoria, en tres grupos de 20 niños cada uno, dos de los cuales recibieron un fármaco (Paracetamol 0 lbuprofeno), administrado 30 minutos antes del acto quirúrgico, y cuya dosis fue de acuerdo al peso de cada paciente: paracetamol $15 \mathrm{mg} / \mathrm{kg}$ e lbuprofeno 10 $\mathrm{mg} / \mathrm{kg}$ y el tercero que siguió el protocolo terapéutico convencional (grupo control), al cual se le administró paracetamol (15 mg/kg), luego de la extracción, al momento del paciente referir dolor. Los pacientes fueron sometidos a la exodoncia de las primeras molares deciduas inferiores (piezas $7,4 \circ 8,4$ ).

Las variables estudiadas fueron: presencia de dolor, tiempo de presencia de dolor, intensidad de dolor, y tiempo transcurrido para analgesia de rescate.

Se realizó el procedimiento quirúrgico, anestesiando de manera infiltrativa, según necesidad con lidocaína al $2 \%$ con epinefrina $1: 100,000$, registrando los datos y el acto quirúrgico en la Ficha de Reporte Operatorio, posteriormente se entregó a los padres la escala visual de dolor de Wong-Baker (Fig. 1), para niños de 3 a 8 años.

Al grupo control y de administración de Paracetamol e Ibuprofeno profiláctico se les dio la indicación de tomar las mismas dosis indicadas anteriormente según peso, cuando se presente un dolor que así lo requiera (dolor moderado $=6$, severo $=8$ o muy severo $=10$ ). En la ficha de Monitoreo del dolor se consignaron el intervalo de necesidad de terapia analgésica de rescate en minutos en los pacientes con profilaxis analgésica. Todos los dientes deciduos fueron extraídos intactos con un mínimo de trauma quirúrgico y sin complicaciones.

Se realizó un control de la herida quirúrgica a los 3 días posteriores a la extracción, y se recogieron las fichas de Monitoreo del dolor.

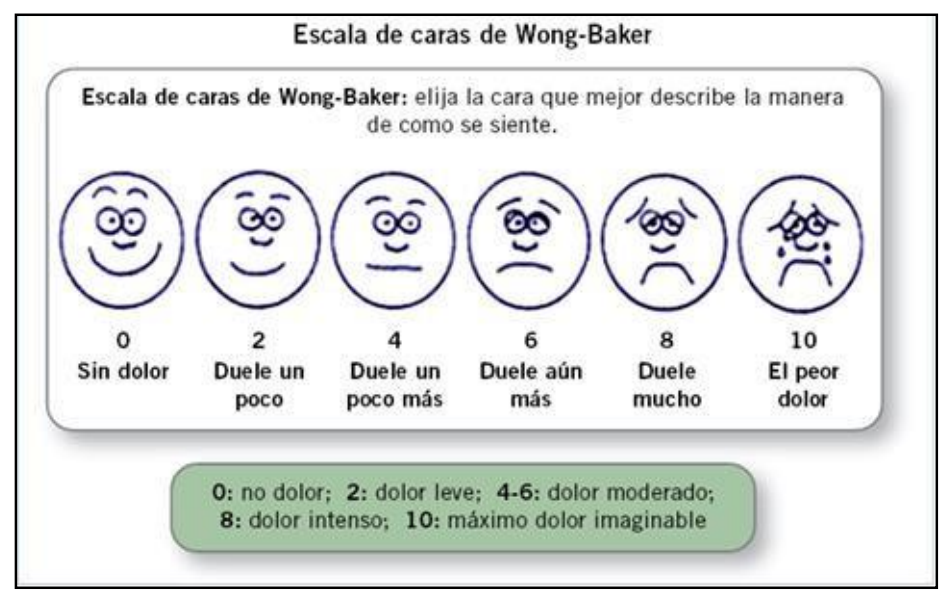

Figura1. Escala visual de Wong Baker 


\section{RESULTADOS}

De acuerdo a las variables intervinientes: edad y sexo; no se encontraron diferencias significativas en el fármaco utilizado en las tres categorías etarias. (Prueba de Chi cuadrado de Pearson $\mathrm{p}=0.970$ ), ni en la variable sexo (Prueba de Chi cuadrado de Pearson $\mathrm{p}=1.00)$.

En cuanto a la presencia de dolor posoperatorio, de los 60 niños pertenecientes a los tres grupos, cada uno con 20 pacientes; se puede observar un valor de significancia de 0.030 , lo que nos indica que si existe relación estadísticamente significativa entre la presencia de dolor y el tipo de terapia instaurada (Tabla 1).
Al evaluarse el tiempo de presencia de dolor posquirúrgico, mediante la prueba estadística paramétrica de Análisis de Varianza de un factor (ANOVA), se encontraron diferencias estadísticamente significativas entre los valores de los tres grupos estudiados (valor de $\mathrm{p}<0.001$ ). (Tabla 2 ).

La tabla 3 muestra los resultados post hoc de comparaciones múltiples de Scheffe en los tres grupos por pares, encontrándose diferencias significativas cuando se compara el tratamiento con Paracetamol frente al grupo control (valor de $\mathrm{p}<0.001$ ), así como al comparar el lbuprofeno con el grupo control (valor de $\mathrm{p}<0.001)$.

Tabla 1. Frecuencia de aplicación de los grupos terapéuticos de acuerdo a la presencia de dolor postoperatorio

\begin{tabular}{|c|c|c|c|c|c|c|c|c|c|}
\hline \multirow[b]{2}{*}{ Dolor } & \multicolumn{2}{|c|}{ paracetamol } & \multicolumn{2}{|c|}{ ibuprofeno } & \multicolumn{2}{|c|}{ Ninguno } & \multicolumn{2}{|c|}{ Total } & \multirow{2}{*}{$\begin{array}{l}\text { Valor de } \\
\text { significanc } \\
\text { ia }\end{array}$} \\
\hline & $\mathbf{n}$ & $\%$ & $\mathbf{n}$ & $\%$ & $\mathbf{n}$ & $\%$ & $\mathrm{n}$ & $\%$ & \\
\hline $\mathrm{Si}$ & 12 & $60.0 \%$ & 9 & $45.0 \%$ & 17 & $85.0 \%$ & 38 & $63.3 \%$ & 0.030 \\
\hline No & 8 & $40.0 \%$ & 11 & $55.0 \%$ & 3 & $15.0 \%$ & 22 & $36.7 \%$ & \\
\hline Total & 20 & $100.0 \%$ & 20 & $100.0 \%$ & 20 & $100.0 \%$ & 60 & $100.0 \%$ & \\
\hline
\end{tabular}

Prueba estadística: Chi Cuadrado de Pearson

Tabla 2. Resultados de comparación del tiempo de presencia de dolor en los tres grupos de estudio

ANOVA

Tiempo de presencia de dolor

\begin{tabular}{lccccc}
\hline & $\begin{array}{c}\text { Suma de } \\
\text { cuadrados }\end{array}$ & gl & $\begin{array}{c}\text { Media } \\
\text { cuadrática }\end{array}$ & $F$ & Sig. \\
\hline Entre grupos & 100081.927 & 2 & 50040.964 & 50.252 & 0.000 \\
Dentro de grupos & 34852.941 & 35 & 995.798 & & \\
Total & 134934.868 & 37 & & & \\
& & & & & \\
\hline
\end{tabular}


Tabla 3. Resultados de la prueba post hoc de Scheffe con las comparaciones múltiples de los grupos evaluados

\section{Comparaciones múltiples}

\begin{tabular}{|c|c|c|c|c|c|c|}
\hline \multicolumn{7}{|c|}{ Tiempo de presencia de dolor } \\
\hline \multirow{2}{*}{\multicolumn{2}{|c|}{ (I) Fármaco administrado }} & \multirow{2}{*}{$\begin{array}{c}\text { Diferencia de } \\
\text { medias (I-J) }\end{array}$} & \multirow[b]{2}{*}{ Desv. Error } & \multirow[b]{2}{*}{ Sig. } & \multicolumn{2}{|c|}{ Intervalo de confianza al $95 \%$} \\
\hline & & & & & Límite inferior & $\begin{array}{l}\text { Límite } \\
\text { superior }\end{array}$ \\
\hline \multirow[t]{2}{*}{ Paracetamol } & ibuprofeno & -30.000 & 13.915 & 0.113 & -65.57 & 5.57 \\
\hline & ninguno & $87,941^{*}$ & 11.898 & 0.000 & 57.53 & 118.36 \\
\hline \multirow[t]{2}{*}{ Ibuprofeno } & paracetamol & 30.000 & 13.915 & 0.113 & -5.57 & 65.57 \\
\hline & ninguno & $117,941^{*}$ & 13.008 & 0.000 & 84.69 & 151.20 \\
\hline \multirow[t]{2}{*}{ Ninguno } & paracetamol & $-87,941^{*}$ & 11.898 & 0.000 & -118.36 & -57.53 \\
\hline & ibuprofeno & $-117,941^{*}$ & 13.008 & 0.000 & -151.20 & -84.69 \\
\hline
\end{tabular}

* La diferencia de medias es significativa en el nivel 0.05 .

Al comparar, independientemente del grupo control, los dos grupos en los que se administró profilácticamente el paracetamol y el ibuprofeno; mediante la prueba $\mathrm{t}$ de Student de muestras independientes, el resultado $(p=0.056)$, indica que no existe suficiente evidencia para afirmar que hay diferencias estadísticamente significativas en el tiempo de presencia de dolor al administrar ibuprofeno versus el paracetamol. La intensidad de dolor presentado en los tres grupos de estudio se muestra en el figura 2, no encontrándose asociación entre ambas variables mediante la Prueba de Chi cuadrado, tomando en consideración los tres grupos $\mathrm{p}=0.075$ y sólo los dos grupos de fármacos $\mathrm{p}=0.339$.

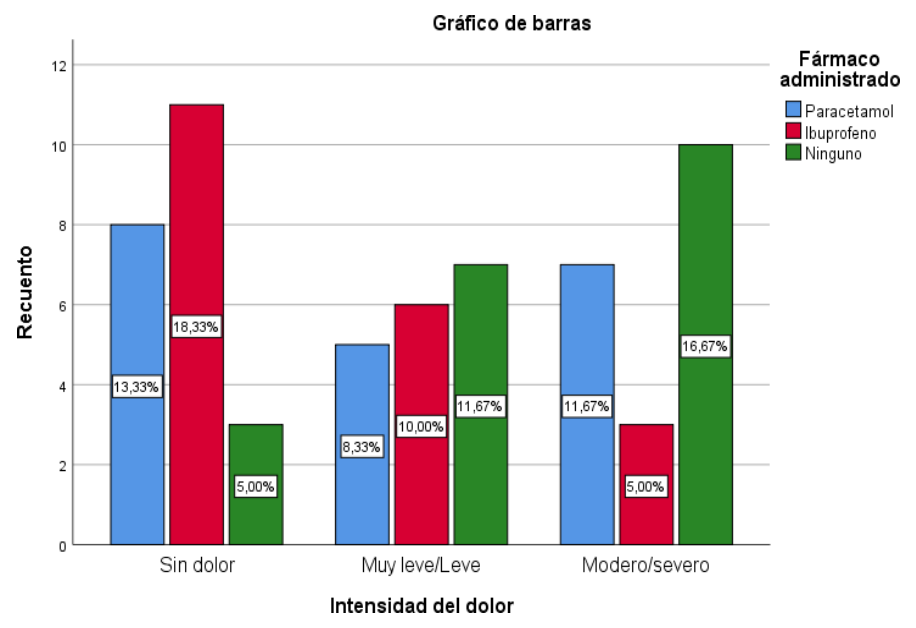

Figura 2. Distribución de la intensidad del dolor de acuerdo a los grupos terapéuticos empleados

La terapia de rescate, administrada a los pacientes que recibieron medicación profiláctica, se presentó en mayor número de casos en el grupo de Paracetamol (7 casos) y en segundo lugar el grupo de lbuprofeno (3 casos), tomando en consideración que no a todos los pacientes que presentaron dolor se les administró medicación posoperatoria, esto dependió de la intensidad del mismo.

Las tablas 4 y 5 muestran los tiempos de terapia analgésica de rescate para cada uno de los fármacos estudiados. Para el tiempo de terapia analgésica de rescate se registró la cantidad de minutos desde que finalizó la cirugía hasta que, de acuerdo a la intensidad del dolor, se le administró al paciente niño la misma medicación dada profilácticamente (grupo paracetamol y grupo ibuprofeno). La tabla 6 muestra los resultados comparando los tiempos de terapia analgésica de rescate de los dos grupos mencionados; la prueba de Levene nos indica que existe homogeneidad de varianzas entre los grupos analizados $(p=0.357)$, el resultado de la prueba estadística t de Student, indica como resultado que no existen diferencias significativas entre ambos medicamentos $(p=0.084)$. 
Tabla 4. Tiempo de terapia analgésica de rescate del paracetamol

\begin{tabular}{|c|c|c|c|}
\hline \multicolumn{4}{|c|}{ Tiempo de terapia analgésica de rescate (min) } \\
\hline \multirow{8}{*}{$\begin{array}{l}\text { Tiempo de rescate } \\
\text { (min) }\end{array}$} & \multicolumn{2}{|l|}{ Media } & \multirow{3}{*}{$\begin{array}{r}180.00 \\
151.12\end{array}$} \\
\hline & $95 \%$ de intervalo de & Límite & \\
\hline & \multirow{3}{*}{\multicolumn{2}{|c|}{$\begin{array}{lll}\begin{array}{l}\text { Confianza } \\
\text { media }\end{array} & \text { para la } \begin{array}{c}\text { inferior } \\
\begin{array}{c}\text { s........ } \\
\text { superior }\end{array}\end{array} \\
\text { Mediana } & & \end{array}$}} & \\
\hline & & & 208.88 \\
\hline & & & 180.00 \\
\hline & \multicolumn{2}{|l|}{ Desv. Desviación } & 31.225 \\
\hline & \multicolumn{2}{|l|}{ Mínimo } & 150 \\
\hline & \multicolumn{2}{|l|}{ Máximo } & 225 \\
\hline
\end{tabular}

Tabla 5. Tiempo de terapia analgésica de rescate del ibuprofeno

\begin{tabular}{|c|c|c|c|}
\hline \multicolumn{4}{|c|}{ Tiempo de terapia analgésica de rescate (min) } \\
\hline \multirow{7}{*}{$\begin{array}{c}\text { Tiempo de rescate } \\
\text { (min) }\end{array}$} & Media & & 220.00 \\
\hline & $\begin{array}{l}95 \% \text { de intervalo de } \\
\text { confianza para lamedia }\end{array}$ & $\begin{array}{l}\text { Límite } \\
\text { inferior }\end{array}$ & 163.08 \\
\hline & & $\begin{array}{l}\text { Límite } \\
\text { superior }\end{array}$ & 276.92 \\
\hline & Mediana & & 225.00 \\
\hline & Desv. Desviación & & 22.913 \\
\hline & Mínimo & & 195 \\
\hline & Máximo & & 240 \\
\hline
\end{tabular}

a. Fármaco administrado = Ibuprofeno

Tabla 6. Tiempo de analgesia de rescate y fármacos administrados

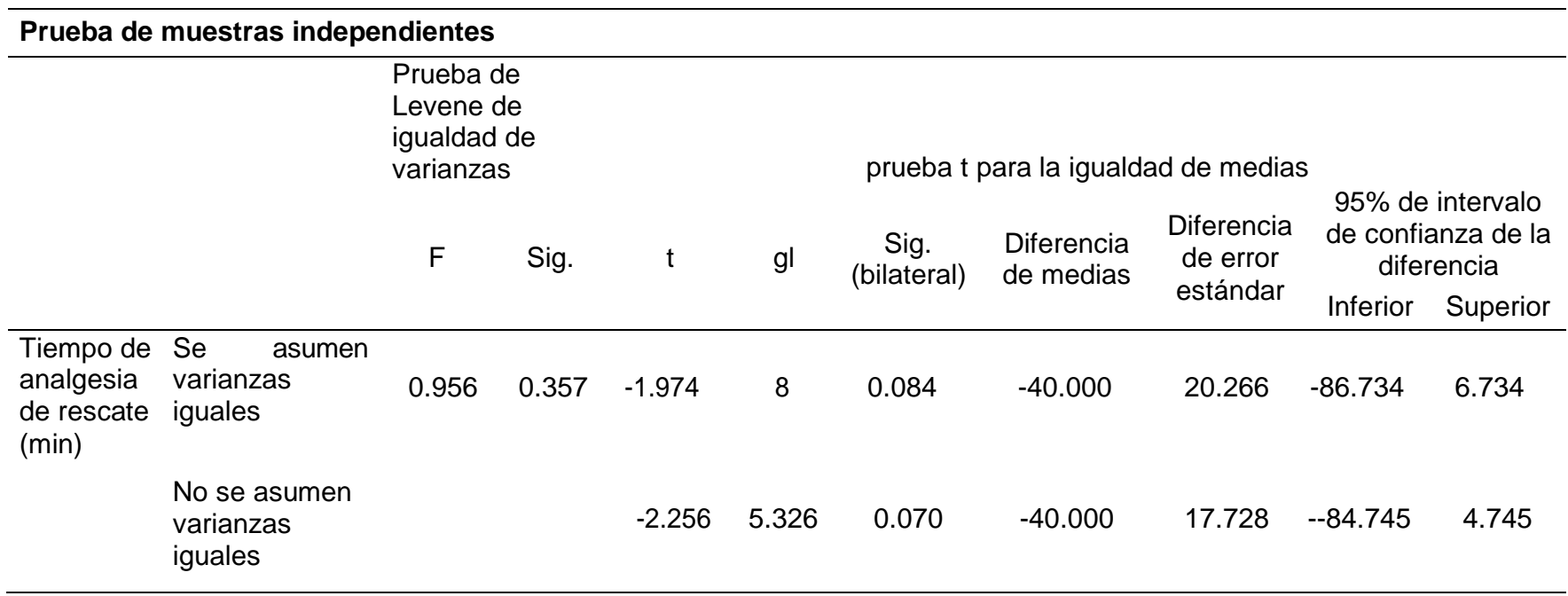




\section{DISCUSIÓN}

El presente estudio comparó el efecto analgésico profiláctico de dos medicamentos comúnmente utilizados en el área pediátrica como tratamiento farmacológico para el dolor y la fiebre: el Paracetamol y el Ibuprofeno.

Uno de los aspectos a evaluar para evitar sesgos fue la edad y el sexo, ya que al tratarse de pacientes niños estas variables hubieran podido influir de alguna forma en la investigación, tomando en cuenta los factores biológicos y cognoscitivos ${ }^{(3,4)}$. De acuerdo a los resultados obtenidos no se encontraron diferencias significativas para ambas variables.

La presencia de dolor posquirúrgico en los tres grupos evaluados presentó diferencias significativas entre ellos $(p=0.030)$, lo que nos indica que la terapia profiláctica analgésica es eficaz en los tratamientos quirúrgicos en niños, siendo el grupo del lbuprofeno el que presentó menos casos de dolor $(n=9)$, seguido por el de Paracetamol $(n=12)$ y finalmente el grupo control que presentó 17 casos de dolor posquirúrgico. Estos resultados contrastan con el estudio realizado por Primosh y colaboradores ${ }^{(5)}$, quienes compararon los mismos fármacos no encontrando diferencias, lo cual podría haberse debido a que la dosis de los medicamentos no se calcularon en base a $\mathrm{mg} / \mathrm{kg}$ de peso, tomándose en cuenta la edad de los niños participantes en el estudio; así como que la administración se dio en un intervalo menor al establecido de acuerdo al mecanismo de acción de los fármacos. Sin embargo en revisiones de estudios sobre profilaxis analgésica en terceras molares se concluye que ésta es un método más exitoso para suprimir el dolor posoperatorio ya que la administración prequirúrgica de un analgésico puede inhibir la sensibilización central por daño tisular ${ }^{(6,7,8)}$ y que si ésta en algunos casos no es demostrada puede deberse a una subdosificación o no dosificación $(9,10)$, como lo demostrado por Milani en un estudio realizado con estos medicamentos (11).

Los tiempos transcurridos para que la presencia de dolor se diera también fueron evaluados en cada grupo, considerando que en algunos pacientes de cada grupo no se presentó dolor posoperatorio; sin embargo en los casos que si se reportó dolor se puede observar que la media en el grupo control fue de 112.06 minutos, tiempo menor al presentado por el Paracetamol con 200 minutos y el lbuprofeno con 230 minutos; esto debido a la acción antiinflamatoria y analgésica previa establecida. Al comparar lavariable tiempo de presencia de dolor entre los tres grupos, mediante ANOVA encontramos que existen diferencias significativas $(p<0.001)$, sin embargo al realizar la comparación por pares solo se encuentran diferencias entre el grupo control con el de paracetamol $(p<0.001)$ y entre el grupo control con el de Ibuprofeno $(p<0.001)$, no encontrándose significancia entre los grupos de paracetamol e Ibuprofeno $(p=0.113)$. Lo que demuestra que la profilaxis analgésica previene o retrasa la aparición del dolor posoperatorio. La analgesia preventiva se basa en que el inicio de la intervención analgésica antes de la nocicepción es más efectivo que su aplicación luego de iniciada ésta y que la duración de la acción farmacológica de la medicación empleada sea mayor; siendo su objetivo prevenir en el sistema nervioso central (SNC) la sensibilización central o los fenómenos que la producen, lo que conduce a la presencia de dolor $(12,13)$.

Los antiinflamatorios no esteroideos (AINES) a menudo se usan para el dolor dental posquirúrgico. La administración postoperatoria de un AINE es efectiva una vez que el fármaco se ha absorbido y distribuido por todo el cuerpo. Actúa inhibiendo la síntesis de prostaglandinas a partir del ácido araquidónico. Al ser administrado un AINE, existe una cantidad de"tiempo de retraso" que puede permitir que comience la inflamación y que el paciente experimente dolor. La administración preventiva de un AINE puede no solo enmascarar el dolor a través de los efectos analgésicos, sino que también evita o disminuye la cascada de mediadores inflamatorios que causa dolor posquirúrgico $(14,15)$.

En la comparación de dos grupos mediante la prueba de $t$ de Student en los que se administró profilácticamente el Paracetamol y el lbuprofeno se halló un valor de $p=0.056$, lo que nos indica que no existen suficiente evidencia para afirmar que existen diferencias estadísticamente significativas en el tiempo de presencia de dolor entre los dos fármacos, a diferencia del estudio que realizó Dionne en donde comparó la terapia profiláctica analgésica del ibuprofeno con el paracetamol en la extracción de terceras molares y concluyó que el tratamiento previo con ibuprofeno retrasa la aparición y suprime la intensidad del dolor postoperatorio en mayor medida que la terapia analgésica con paracetamol o la tradicional sin un aumento en los efectos secundarios (16). La mayor eficacia del tratamiento previo con ibuprofeno en comparación con estos analgésicos estándar sugiere que la supresión de los procesos que contribuyen al dolor postoperatorio, es decir, la cascada de ácido araquidónico, produce menos dolor que la administración postoperatoria de fármacos que intentan aliviar el dolor mediante el antagonizar las vías de dolor activadas $(17,18)$.

El ibuprofeno ha demostrado en diferentes investigaciones que al ser administrado inmediatamente antes de la cirugía o inmediatamente 
posterior a ésta, retrasó significativamente la aparición de dolor en comparación con un placebo (19). Asimismo si se toma ibuprofeno antes de la cirugía, se esperaría que la concentración plasmática esté por debajo de los niveles óptimos de 5 a 6 horas después de la administración. Cuando el Ibuprofeno se toma después de la operación, los niveles plasmáticos máximos se alcanzan en 1 a 2 horas después de la administración (20). En estudios realizados que no implican actos quirúrgicos como el de Steen Law, de la especialidad de ortodoncia, el Ibuprofeno confirmó su acción analgésica preventiva al ser administrado 1 hora antes de la cita de ortodoncia para la colocación del separador, donde los pacientes refirieron tener mucho menos dolor al masticar que los pacientes que recibieron lbuprofeno después de la operación o un placebo ${ }^{(21)}$.

El Paracetamol es un analgésico no opioide que posee actividad antipirética y es eficaz para aliviar el dolor con una baja incidencia de efectos adversos. En la actualidad el paracetamol se agrupa con la familia de medicamentos antiinflamatorios no esteroides (AINES), considerándosele un inhibidor selectivo de una variante de la ciclooxigenasa-1: la enzima COX3. Posee una actividad antiinflamatoria relativamente débil en el sistema nervioso central; sin embargo, se considera que esta inhibición podría representar un mecanismo central primario mediante el cual el Paracetamol disminuye el dolor y posiblemente la fiebre. Se ha demostrado que es un analgésico eficaz en el control del dolor dental postoperatorio en varios ensayos clínicos ${ }^{22,23)}$.

La intensidad de dolor, en los pacientes que lo presentaron, fue evaluada en los tres grupos encontrándose que el grupo control presentó el mayor número de casos con dolor de mayor intensidad $(n=10)$, seguidos del grupo del paracetamol $(n=7)$ y el del ibuprofeno $(n=3)$, a pesar de estas diferencias no se encontró significancia entre los tres grupos e intensidad de dolor $(p=0.075)$.

Al realizar la asociación entre el fármaco administrado (Paracetamol e Ibuprofeno) y la intensidad de dolor producida luego del acto operatorio, no se halló estadísticas significativas entre ambas variables $(p=0.339)$. Estos resultados difieren de los presentados por otros investigadores (24) quienes realizaron profilaxis analgésica con dichos fármacos en exodoncias de terceras molares, demostrando que los casos a los que se les administró Ibuprofeno presentaban nivel de dolor significativamente menores, lo que puede haberse debido a que los niveles de dolor presentados en ese tipo de cirugías pueden ser bastante más elevados que en piezas deciduas, lo que permite realizar una diferenciación mayor de éstos.
Asimismo, hay que tener en cuenta que el dolor es multidimensional e incluye nocicepción, percepción y expresión. Por esta razón, se deben considerar múltiples aspectos de la experiencia del dolor, incluidas las dimensiones sensoriales, afectivas y cognitivas. No existe un enfoque único para la evaluación del dolor que sea apropiado para todos los pacientes o en todos los entornos porque la naturaleza de la evaluación se ve afectada por múltiples factores, incluido el propósito de la evaluación, el entorno en el que se realiza la evaluación, la población de pacientes y el clínico ${ }^{(25)}$, más aún cuando nuestra población es infantil.

Una revisión de Jackson et al. (26) mostró que la administración preoperatoria de AINES puede retrasar la aparición del dolor, disminuir los niveles de dolor y retrasar la intensidad máxima del dolor, lo que se demuestra en este estudio al comparar los casos que presentaron dolor y el nivel de intensidad del mismo.

Los tiempos de terapia analgésica de rescate de los dos grupos paracetamol e ibuprofeno fueron comparados, obteniéndose como resultado que no existen diferencias significativas entre ambos medicamentos $(p=0.084)$.

La mayoría de estudios en niños, con analgesia preventiva ha sido realizada en cirugías otorrinolaríngeas: amidalectomías y adenoidectomias, donde el Ibuprofeno ha demostrado superioridad al Paracetamol en la disminución del dolor de la cirugía posoperatoria, dando como resultado un mayor efecto analgésico en comparación con los tratamientos postoperatorios estándar y otros AINES combinados con opiáceos utilizados antes de la operación (27, 28, 29).

El presente estudio concluyó que el efecto analgésico profiláctico en la variable presencia de dolor del Paracetamol y el Ibuprofeno resultó más eficaz que el tratamiento farmacológico convencional posquirúrgico utilizado en niños, asimismo el tiempo de presencia de dolor fue menor en el grupo control en comparación con los grupos con fármacos, lo que demuestra que el uso de AINES, de uso común en pediatría, antes de un acto quirúrgico odontológico en niños, disminuye la presencia de dolor posquirúrgico, así como su tiempo de aparición e intensidad.

Al realizar la comparación de los dos fármacos: paracetamol e ibuprofeno no existen diferencias significativas que nos demuestren que uno es superior al otro, para ser utilizado como elección en la profilaxis analgésica.

Contribuciones de autoría: MPT diseñó el estudio, recopiló los datos, analizó los resultados y redactó el artículo. RMV brindo asesoría estadística. Ambos autores aprobaron la versión final del artículo. 
Fuente de financiamiento: El estudio fue financiado por la autora MPT.

Conflicto de intereses: Los autores declararon no tener conflictos de interés.

\section{REFERENCIAS}

1. IASP, 1994. Part III: (pp 209-214). Classification of Chronic Pain, Second Edition, IASP Task Force on Taxonomy, edited by $\mathrm{H}$. Merskey and N. Bogduk, ISAP Press, Seattle, 1994. http://www.iasp- pain.org.

2. González de Mejía N.: Postoperative multimodal analgesia. Rev Soc Esp Dolor, 2005; 12:112-118.

3. Tovar MA. Dolor en niños. Colombia Médica. 2005; 36(4): 62-68.

4. Hartling L, Ali S, Dryden DM, Chordiya P, Johnson DW, Plint AC, Stang A, McGrath PJ, Drendel AL, How Safe Are Common Analgesics for the Treatment of Acute Pain for Children? A Systematic Review. Pain Res Manag. 2016; 2016:5346819.

5. Primosch RE, Nichols DL, Courts FJ, Comparison of preoperative ibuprofen, acetaminophena and placebo administration on the parental report of postextraction pain in children. Pediatr Dent.1995; 17(3):187-91

6. Chumpitaz Cerrate V. Comparación de Diclofenaco y Ketorolaco para prevenir el dolor posterior a cirugía de tercera molar retenida [Tesis para optar el grado de Magister en Farmacología]. Lima: Facultad de Farmacia y Bioquímica, Universidad Nacional Mayor de San Marcos; 2007.

7. Esquivel Velásquez G. Efectividad del diclofenaco y Ketoprofeno como profilaxis analgésica en cirugía de terceras molares retenidas [Tesis para optar el título de Cirujano Dentista]. Lima: Facultad de Odontología, Universidad Nacional Mayor de San Marcos; 2009.

8. Yamaguchi A, Sano K, Effectiveness of preemptive analgesia on postoperative pain following third molar surgery: Review of literatures. Japan Dent Science Review. 2013; 49(4): 131-138

9. Goodman Gilman A. Las bases farmacológicas de la terapéutica. $8^{\circ}$ ed. Buenos Aires. Médica Panamericana. 1991.

10. Litter Manuel. Compendio de farmacología. $5^{\circ}$ ed. Buenos Aires. El Ateneo. 2001.

11. Milani GP, Benini $F$, Dell'Era L, Silvagni $D$, Podestà AF, Mancusi RL, Fossali Ef; Pierre GROUP STUDY. Acute pain management: acetaminophen and ibuprofen are often under- dosed. Eur J Pediatr. 2017; 176(7):979-982.

12. Woolf $\mathrm{C}$, Chong M. Preemptive analgesia - treating postoperative pain by preventing the establishment of central sensitization. Anesth Analg 1993; 77:362-79.

13. McQuay $H$, Dickenson A. Implications of nervous system plasticity for pain management. Anaesthesia 1990; 45:101-2.

14. Paracetamol for pain relief after surgical removal of lower wisdom teeth (Protocol) Weil K, Afzal Z, Esposito M, Worthington HV, van Wijk AJ, Hooper L, Coulthard P. Cochrane Database Syst Rev. 2007; 18(3): CD004487

15. Attar S1, Bowles WR, Baisden MK, Hodges JS, McClanahan SB. Evaluation of pretreatment analgesia and endodontic treatment for postoperative endodontic pain. J Endod. 2008; 34(6):652-5

16. Dionne R, Campbell R, Cooper S, Hall D, Buckingham $\mathrm{B}$, Suppression of Postoperative Pain by Preoperative
Administration of Ibuprofen in Comparison to Placebo, Acetaminophen and Acetaminophen plus Codeine. J Clin Pharmacol. 1983; 23:37-43.

17. Viitanen $\mathrm{H}$, Tuominen N, Nikanne E, Annila P, Analgesic eficacy of rectal acetaminophen and ibuprofen alone or in combination for paediatric day-case adenoidectomy. British Journal of Anaesthesia 2003; 91(3): 363-7.

18. Charles $C$, Matt B, Hamilton M, Katz B, A comparison of ibuprofen versus acetaminophen with codeine in the young tonsillectomy patient. Otolaryngol Head Neck Surg 1997; 117:76-82.

19. Lesko SM1, Mitchell AA, An assessment of the safety of pediatric ibuprofen. A practitioner-based randomized clinical trial. JAMA. 1995; 273(12):929-33.

20. Vogel R, Desjardins J, Major K, Comparison of Presurgical and Immediate Postsurgical Ibuprofen on Postoperative Periodontal Pain. J Periodontol. 1992; 63(11):914-18.

21. Steen Law SL, Southard KA, Law AS, Logan HL, Jakobsen JR. An evaluation of preoperative ibuprofen for treatment of pain associated with orthodontic separator placement. Am J Orthod Dentofacial Orthop. 2000 Dec;118(6):629-35.

22. Moore RA, Derry S, Wiffen PJ, Straube S, Aldington DJ. Overview review: Comparative efficacy of oral Ibuprofen and Paracetamol (acetaminophen) across acute and chronic pain conditions. Eur J Pain. 2015; 19(9):1213-23.

23. Kanabar DJ, A clinical and safety review of Paracetamol and Ibuprofen in children. Inflammopharmacology. 2017;25(1):1-9.

24. Ashley, P.F., Parekh, S., Moles, D.R., Anand, P., Behbehani, A. Preoperative analgesics for additional pain relief in children and adolescents having dental treatment. Cochrane Database Syst Rev. 2016; (8):CD008392

25. Welchek $\mathrm{C}$, Mastrangelo $\mathrm{L}$, Sinatra $\mathrm{R}$, Martinez $\mathrm{R}$, Qualitative and Quantitative Assessment of Pain. En Sinatra R. Acute Pain Mangement. Cambridge University Press, 2009. p.147-171

26. Jackson D, Moore P, Hargreaves K. Preoperative nonsteroidal anti-inflammatory medication for the prevention of postoperative dental pain. J Am Dent Assoc 1989;119:641-7.

27. Shirvani A, Shamszadeh S, Eghbal MJ, Marvasti LA, Asgary S. Effect of preoperative oral analgesics on pulpal anesthesia in patients with irreversible pulpitis-a systematic review and meta-analysis. Clin Oral Investig. 2017 Jan;21(1):43-52.

28. Liu C1, Ulualp SO, Outcomes of an Alternating Ibuprofen and Acetaminophen Regimen for Pain Relief After Tonsillectomy in Children. Ann Otol Rhinol Laryngol. 2015;124(10):777-81.

29. Merry AF, Edwards KE, Ahmad Z, Barber C, Mahadevan M, Frampton C, Randomized comparison between the combination of Acetaminophen and Ibuprofen and each constituent alone for analgesia following tonsillectomy in children. Can J Anaesth. 2013; 60(12):1180-9. 